\title{
Predicting genome-scale Arabidopsis- Pseudomonas syringae interactome using domain and interolog-based approaches
}

\author{
Sitanshu S Sahu ${ }^{1,2}$, Tyler Weirick ${ }^{1,2}$, Rakesh Kaundal ${ }^{3^{*}}$ \\ From 11th Annual MCBIOS Conference \\ Stillwater, OK, USA. 6-8 March 2014
}

\begin{abstract}
Background: Every year pathogenic organisms cause billions of dollars' worth damage to crops and livestock. In agriculture, study of plant-microbe interactions is demanding a special attention to develop management strategies for the destructive pathogen induced diseases that cause huge crop losses every year worldwide. Pseudomonas syringae is a major bacterial leaf pathogen that causes diseases in a wide range of plant species. Among its various strains, pathovar tomato strain DC3000 (PstDC3000) is asserted to infect the plant host Arabidopsis thaliana and thus, has been accepted as a model system for experimental characterization of the molecular dynamics of plant-pathogen interactions. Protein-protein interactions (PPIs) play a critical role in initiating pathogenesis and maintaining infection. Understanding the PPI network between a host and pathogen is a critical step for studying the molecular basis of pathogenesis. The experimental study of PPIs at a large scale is very scarce and also the high throughput experimental results show high false positive rate. Hence, there is a need for developing efficient computational models to predict the interaction between host and pathogen in a genome scale, and find novel candidate effectors and/or their targets.
\end{abstract}

Results: In this study, we used two computational approaches, the interolog and the domain-based to predict the interactions between Arabidopsis and PstDC3000 in genome scale. The interolog method relies on protein sequence similarity to conduct the PPI prediction. A Pseudomonas protein and an Arabidopsis protein are predicted to interact with each other if an experimentally verified interaction exists between their respective homologous proteins in another organism. The domain-based method uses domain interaction information, which is derived from known protein 3D structures, to infer the potential PPIs. If a Pseudomonas and an Arabidopsis protein contain an interacting domain pair, one can expect the two proteins to interact with each other. The interolog-based method predicts $\sim 0.79 \mathrm{M}$ PPIs involving around 7700 Arabidopsis and 1068 Pseudomonas proteins in the full genome. The domain-based method predicts 85650 PPIs comprising 11432 Arabidopsis and 887 Pseudomonas proteins. Further, around 11000 PPIs have been identified as interacting from both the methods as a consensus.

Conclusion: The present work predicts the protein-protein interaction network between Arabidopsis thaliana and Pseudomonas syringae pv. tomato DC3000 in a genome wide scale with a high confidence. Although the predicted PPIs may contain some false positives, the computational methods provide reasonable amount of interactions which can be further validated by high throughput experiments. This can be a useful resource to the plant community to characterize the host-pathogen interaction in Arabidopsis and Pseudomonas system. Further, these prediction models can be applied to the agriculturally relevant crops.

\footnotetext{
* Correspondence: rkaundal@ucr.edu

${ }^{3}$ Bioinformatics Facility, Department of Botany \& Plant Sciences, Institute for Integrative Genome Biology (IIGB), University of California, Riverside,

California, 92521, USA

Full list of author information is available at the end of the article
}

(c) 2014 Sahu et al.; licensee BioMed Central Ltd. This is an Open Access article distributed under the terms of the Creative Commons Attribution License (http://creativecommons.org/licenses/by/4.0), which permits unrestricted use, distribution, and reproduction in any medium, provided the original work is properly cited. The Creative Commons Public Domain Dedication waiver (http:// creativecommons.org/publicdomain/zero/1.0/) applies to the data made available in this article, unless otherwise stated. 


\section{Background}

Pseudomonas syringae is a Gram-negative bacterium causing economically important diseases in a wide range of plant species leading to severe agricultural losses worldwide. Each strain of Pseudomonas shows a high degree of host specificity and infects only a limited number of plant species or even a few cultivars of a single plant species $[1,2]$. Among them, pathovar tomato strain DC3000 (Pst DC3000) has been asserted to infect the plant host Arabidopsis thaliana and tomato causing bacterial spec and brown spot. Thus, Arabidopsis-Pseudomonas has been accepted as a model system for experimental characterization of the molecular dynamics of plant-pathogen interactions in both resistance and susceptible interactions $[1,3,4]$. The whole genome sequence of Pst DC3000 revealed that it has $\sim 300$ virulence-related genes [5]. One of the major classes of virulence factors includes effector proteins that are delivered into the host through a type III protein secretion system (TTSS) to suppress plant immune responses, and also to facilitate disease development [6]. Basically, Pseudomonas syringae pathogenesis is dependent on effector proteins and to date, nearly 60 different type III effector proteins encoded by hop genes have been identified [http://www.pseudomonas-syringae.org/]. In addition, Pst DC3000 also produces non-proteinaceous virulence effectors, including coronatine (COR), which are crucial for pathogenesis. However, the virulence function of a large number of potential effectors encoded by the Pst DC3000 genome and their mode of action is still unknown. Similarly, in Arabidopsis it has been seen that approximately 3000 proteins are directly related to plant defense [7]. Many of these proteins interact directly with the pathogen proteins and some of them initiate plant defense responses to the infection. Recently, Mukhtar et al. [8] reported an experimental protein interaction network (PPIN-1) containing 843 Arabidopsis proteins and 83 pathogen effectors including very few interactions with Pst DC3000. Till now, only nearly $10 \%$ of the full genome of Arabidopsis has been evidenced for interaction. Therefore, to functionally characterize the dynamic interactions of plants with bacterial pathogens, there is a need for genome-wide study of the host-pathogen interactions. Knowledge of such novel resistance interactions provides the backbone of the understanding of plant resistance mechanisms and will aid in the further analysis of plant immunity [9].

Generally, pathogen attacks host tissues, secreting degradation enzymes and toxin release. Many of such mechanisms involve the protein-protein interactions (PPIs). PPIs are essential process in all living cells and play a crucial role in the infection process, and initiating a defense response. In this context, understanding the PPI network (interactome) between plant proteins and pathogen proteins is a critical step for studying the molecular basis of pathogenesis $[10,11]$. In particular, computational approaches ameliorate the study of hostpathogen protein interactions in a genome-wide range.

In the past decade, a series of PPI prediction methods have been elegantly developed and are playing an increasingly important role in complementing experimental approaches. Diverse data types or properties, such as gene ontology (GO) annotations [12], protein sequence similarity [13], protein domain interactions [14], and protein structural information $[15,16]$ have been frequently utilized to construct PPI prediction methods. Among these computational methods, the interolog and the domainbased methods [17-23] are widely used approaches for PPIs prediction.

In this work, we used the interolog and the domainbased methods to jointly predict the protein-protein interactions between Pseudomonas syringae and Arabidopsis thaliana. The domain-based approach infers inter-species protein-protein interactions by known domain-domain interactions from various databases and the interolog approach identifies protein-protein interactions based on homologous pairs of protein interactions across different organisms. We present the prediction pipeline in detail and the functional analysis of the predicted results.

\section{Materials and methods Data sources}

The whole proteome of Pseudomonas syringae pv. tomato DC3000 is downloaded from Pseudomonas genome database (http://www.pseudomonas.com/download.jsp) which contains 5619 protein sequences. Similarly, the full genome of Arabidopsis thaliana containing 35386 protein sequences is extracted from the TAIR10 database (http://www.arabidopsis.org/). To infer the prediction from the interolog, we have used two types of datasets: the HPIDB dataset and DIP dataset. Database of Interacting Proteins (DIP) is a collection of experimental determined interactions between proteins in intra-species [24]. As of Jan 2014, DIP database contains 25749 sequences of 72380 protein-protein interactions. Host Pathogen Interaction Database (HPIDB) is a database of experimental determined interactions between 62 host and 529 pathogens [25]. As of Jan 2014, HPIDB database contains 29922 sequences of 23735 unique protein-protein interactions. To implement the domain based model, the domain-domain interaction databases, iPfam and 3DID are used. The iPfam database is a catalog of protein family interactions, including domain and ligand interactions, calculated from known structures in protein data bank (PDB). As of Jan 2014, the iPfam1.0 database contains 5442 domain-domain interactions. The database of three-dimensional interacting domains (3DID) is a collection of high-resolution three-dimensional structural templates for domain-domain interactions. It contains 
templates for interactions between two globular domains as well as novel domain-peptide interactions. As of Jan. 2014, the 3DID database contains 8323 domain-domain interactions.

\section{Identification of secreted proteins in Pseudomonas syringae}

All proteins of Pseudomonas are processed through the Psortb3.0 (widely used tool for protein localization in bacteria [26]) and those predicted as cytoplasmic or cytoplasmic membrane are discarded as these proteins have less chance of involvement in interaction. The rest proteins annotated with extracellular, outer membrane and unknown are considered to be positive candidates for interaction. Again we search the whole proteome of Pseudomonas through the effector database (http://www.effectors.org/) [27], which is an integrated database for secreted type proteins for bacteria. Those identified as secreted are considered as positive candidates for interaction. Combining these two steps, 2744 potential candidate proteins of PstDC3000 are filtered for interaction prediction.

\section{Prediction of PPIs between Arabidopsis and Pseudomonas} In this study, the probability of interaction between an Arabidopsis and a Pseudomonas protein is inferred from two approaches: the domain based and the interolog method individually. The prediction framework is shown in Figure 1.

\section{Domain based protein-protein interaction prediction}

The domain-based method uses domain interaction information, which is derived from known protein 3D structures, to infer the potential PPIs. If two proteins contain an interacting domain pair, it is expected that these two proteins may interact with each other. To get the domains in
Arabidopsis and Pseudomonas, HMMPfam is used in interproscan 5 [28]. In total, 49073 domains are extracted for all the Arabidopsis proteins and 7253 domains are collected for PstDC3000. If a protein pair between Pseudomonas and Arabidopsis contains an interacting domain pair from iPfam and 3DID, then the pair is expected to interact with each other.

\section{Interolog based protein-protein interaction prediction}

The interolog method relies on protein sequence similarity to conduct the PPI prediction. An interolog is a conserved interaction between a pair of proteins which have interacting homologs in another organism [29]. The illustration of interolog is shown in Figure 2. Consider that $A$ and $B$ are two different interacting proteins of one organism, and $A^{\prime}$ and $B$ ' are two different interacting proteins of another organism. Then the interaction between $A$ and $B$ is an interolog of the interaction between $A^{\prime}$ and $B^{\prime}$, if $A$ is a homolog of $A^{\prime}, B$ is a homolog of $B^{\prime}, A$ and $B$ interact, and $A^{\prime}$ and $B^{\prime}$ interact. Thus, interologs are homologous pairs of protein interactions across different organisms. Each protein in Arabidopsis and Pseudomonas is BLASTed against all the protein sequences in the DIP and HPIDB database to identify the homologs with E-value, sequence identity and aligned sequence length coverage of $1.0 \mathrm{E}^{-4}, 50$ and $80 \%$ respectively. Each protein pair between Pseudomonas and Arabidopsis is predicted to interact if an experimentally verified interaction exists between their respective homologous proteins in DIP or HPIDB databases.

\section{Results and discussion}

Prediction of interactions

To predict the genome wide interactions, all proteins of Arabidopsis and Pseudomonas are paired up, which constitute $\sim 97 \mathrm{M}$ PPIs. The interaction probability of each

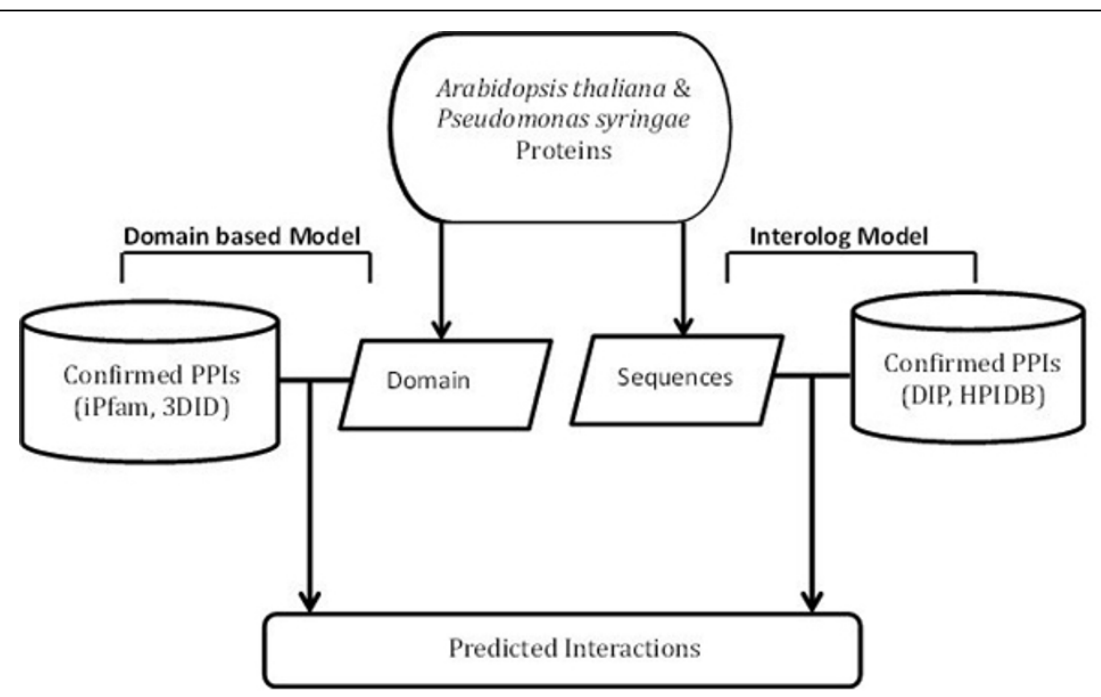

Figure 1 Overall prediction framework of the interactions between Arabidopsis thaliana and Pseudomonas syringae. 


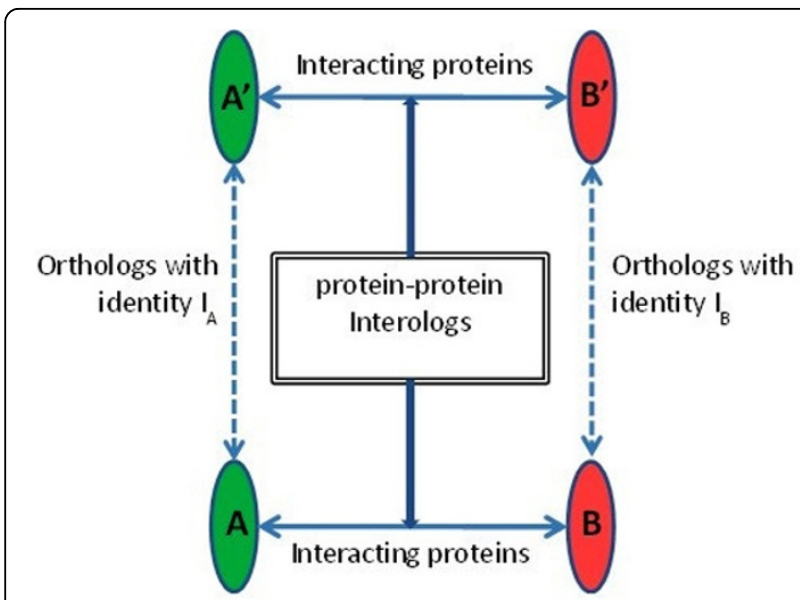

Figure 2 Illustration of protein-protein interologs. $A$ and $B$ are two different interacting proteins in one organism and $A^{\prime}$ and $B^{\prime}$ are two interacting proteins in another organism. Protein $A-A^{\prime}$ and $B-B^{\prime}$ are orthologs between the two organisms. Thus, protein pair $A^{\prime}-B^{\prime}$ and $A-B$ are interologs and conserved in the organisms.

pair is assessed through the domain-based model and interolog-based model separately. The predicted interactions from these methods are reported in Table 1. A total of $\sim 0.86 \mathrm{M}$ probable PPIs are predicted from both the methods, which include $\sim 14043$ Arabidopsis proteins and 1337 Pesudomonas proteins. Out of these, 85650 PPIs are predicted by domain based method involving 11432 Arabidopsis and 887 Pseudomonas proteins. Similarly, the interolog method predicted $\sim 0.79 \mathrm{M}$ PPIs including 7766 Arabidopsis and 1068 Pseudomonas proteins. Nearly, 11000 PPIs are consistently predicted by both methods as consensus which comprises 2043 Arabidopsis and 93 Pseudomonas proteins. The interaction network of the consensus predicted PPI is shown in Figure 3. On average, a Pseudomonas protein has around 118 Arabidopsis interacting partners, whereas an Arabidopsis protein interact with around 6 Pseudomonas proteins. The reported results are coherent with the previous studies in which it is demonstrated that a few pathogen proteins involved in interaction in the host interactome $[11,18,19]$. All predicted interactions from the domain based method, interolog method and the consensus predictions are available in Tables S1-S3 respectively in Additional files 1, 2 and 3.

\section{Predicted effector hubs}

The effectors of Pseudomonas with the highest number of edges (hubs) are PSPTO_0135, PSPTO_0400, PSPTO_0540, PSPTO_0808, PSPTO_1510, PSPTO_2303, PSPTO_2529, PSPTO_2632, PSPTO_3161, PSPTO_3583, PSPTO_3890, PSPTO_3912 and PSPTO_4001 with more than 400 PPIs in the Arabidopsis-Pseudomonas interactome. There are also several effectors with more than 40 predicted PPIs. These are PSPTO_4497, PSPTO_1482, PSPTO_4868, PSPTO_4602, PSPTO_3882, PSPTO_0405, PSPTO_1492, PSPTO_4093, PSPTO_1949, PSPTO_4776, PSPTO_3130, PSPTO_3900, PSPTO_5014 and PSPTO_4090. In contrast to these hub proteins, several effectors are predicted to interact with very few proteins. These hub proteins play important role in pathogenesis, hence can be further investigated for deciphering virulence mechanism.

\section{Functional enrichment analysis of proteins involved in the Interaction}

Functional enrichment analysis is an important assessment for elucidating the functional relevance of the host and pathogen proteins involved in the PPIs. The presence of enriched (over-represented) functional categories that are closely related to host defense and pathogen infection support the validity of the predicted PPIs of the prediction models. Gene ontology (GO) is a comprehensive functional system to annotate the gene products. We used the biological process GO term enrichment to see the relevance of the predicted proteins. The Database for Annotation, Visualization and Integrated Discovery (DAVID) is used to conduct the enrichment analysis[30]. The over represented biological processes of Arabidopsis and Pseudomonas proteins in the predicted PPIs are listed in Tables 2 and 3 respectively. The enrichment analysis in Arabidopsis shows that many proteins involved in the biological process, response to cadmium ion and metal ion. In literature, it has been shown that metal ions are required for pathogen virulence and plant defense [31,32]. Fones et al. demonstrated $\mathrm{Zn}, \mathrm{Ni}$ or $\mathrm{Cd}$ are accumulated when Thlaspi caerule resist to a leaf spot caused by Pseudomonas syringae pv. maculicola [31]. Block and James reveal that the plant immune responses include deposition of lignin and callose in the cell wall and production of reactive oxygen species and anti-microbial compounds [33]. Qiu et al. [34] show that

Table 1 Prediction results of Arabidopsis and Pseudomonas syringae interactions using domain and Interolog approaches

\begin{tabular}{lccc}
\hline \multicolumn{1}{c}{ Predicted PPIs } & \# of PPIs & \# Arabidopsis protein involved & \# of Pseudomonas proteins involved \\
\hline Domain based method & 85650 & 11432 & 887 \\
\hline Inerolog method & 794006 & 7766 & 1068 \\
\hline From Both Methods & 868645 & 14043 & 1337 \\
\hline Consensus Interactions & 11011 & 2043 & 93 \\
\hline
\end{tabular}




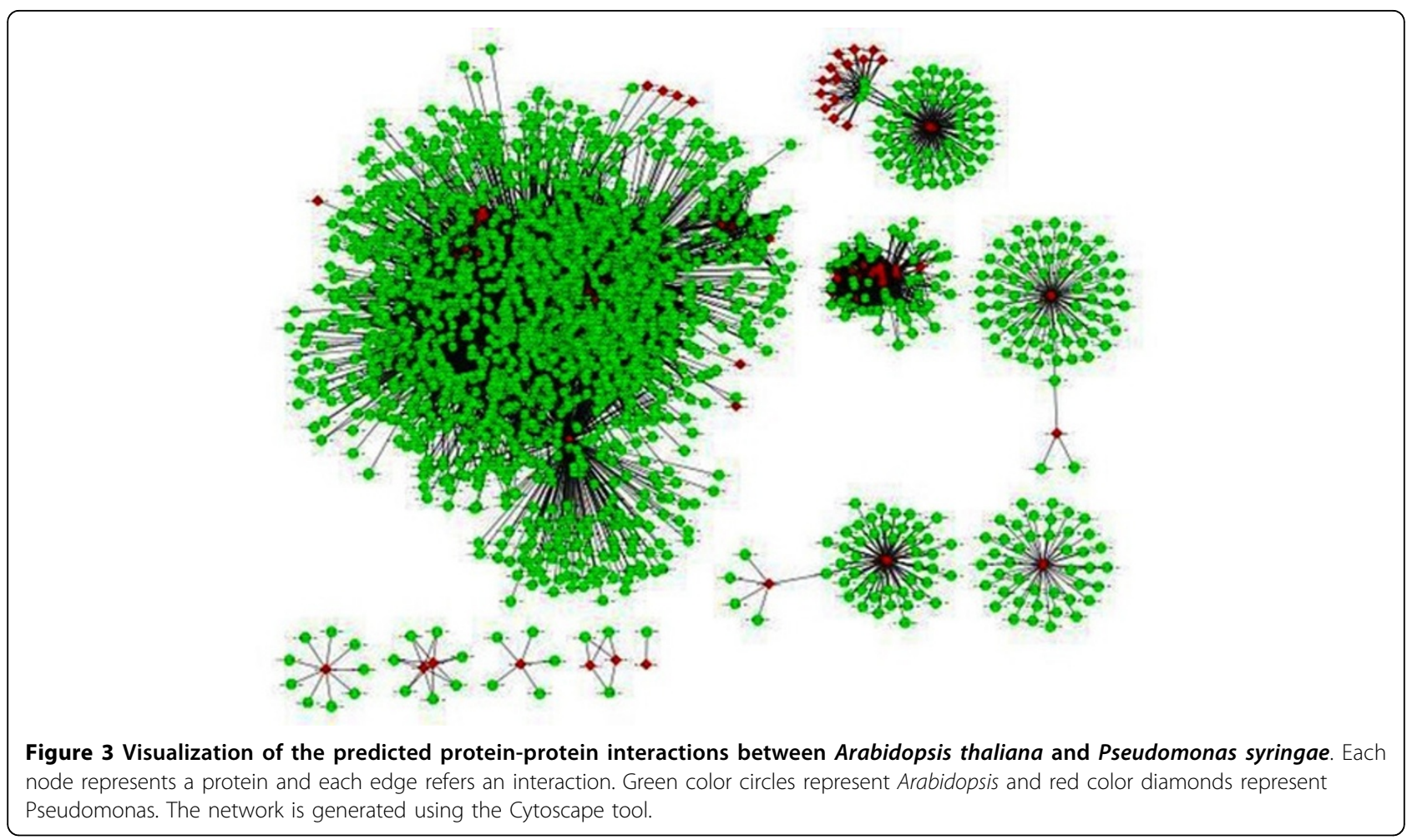

Table 2 Enriched GO biological process terms involved in predicted Arabidopsis proteins

\begin{tabular}{|c|c|c|}
\hline GO id & GO term & $p$-value \\
\hline GO:0006468 & protein amino acid phosphorylation & $8.24 \mathrm{E}-96$ \\
\hline GO:0001906 & cell killing & $5.62 \mathrm{E}-28$ \\
\hline GO:0006796 & phosphate metabolic process & 3.17E-45 \\
\hline GO:0007169 & transmembrane receptor protein tyrosine kinase signaling pathway & $3.16 \mathrm{E}-56$ \\
\hline GO:0007166 & cell surface receptor linked signal transduction & $1.39 \mathrm{E}-67$ \\
\hline GO:0008037 & cell recognition & 1.57E-22 \\
\hline GO:0048610 & reproductive cellular process & $2.39 \mathrm{E}-14$ \\
\hline GO:0015031 & protein transport & $1.69 \mathrm{E}-02$ \\
\hline GO:0007264 & small GTPase mediated signal transduction & $1.34 \mathrm{E}-25$ \\
\hline GO:0007242 & intracellular signaling cascade & $5.98 \mathrm{E}-11$ \\
\hline GO:0009738 & abscisic acid mediated signaling & $9.12 \mathrm{E}-07$ \\
\hline GO:0009755 & hormone-mediated signaling & 2.93E-07 \\
\hline GO:0044070 & regulation of ion transport & $1.62 \mathrm{E}-04$ \\
\hline GO:0032412 & regulation of ion transmembrane transporter activity & $1.74 \mathrm{E}-03$ \\
\hline GO:0009788 & negative regulation of abscisic acid mediated signaling & $4.72 \mathrm{E}-03$ \\
\hline GO:0045454 & cell redox homeostasis & 5.59E-04 \\
\hline GO:0006470 & protein amino acid dephosphorylation & $3.46 \mathrm{E}-18$ \\
\hline GO:0010119 & regulation of stomatal movement & 5.42E-06 \\
\hline GO:0010304 & PSII associated light-harvesting complex II catabolic process & 3.93E-04 \\
\hline GO:0007243 & protein kinase cascade & $6.90 \mathrm{E}-04$ \\
\hline GO:0000165 & MAPKKK cascade & 8.64E-04 \\
\hline GO:0046686 & response to cadmium ion & $3.22 \mathrm{E}-09$ \\
\hline GO:0010038 & response to metal ion & $2.54 \mathrm{E}-06$ \\
\hline
\end{tabular}


Table 2 Enriched GO biological process terms involved in predicted Arabidopsis proteins (Continued)

\begin{tabular}{lll}
\hline GO:0009651 & response to salt stress & $3.76 \mathrm{E}-09$ \\
\hline GO:0009628 & response to abiotic stimulus & $6.03 \mathrm{E}-04$ \\
\hline GO:0009407 & toxin catabolic process & $5.16 \mathrm{E}-11$ \\
\hline GO:0045454 & cell redox homeostasis & $2.37 \mathrm{E}-06$ \\
\hline GO:0016998 & cell wall macromolecule catabolic process & $1.62 \mathrm{E}-05$ \\
\hline GO:0006469 & negative regulation of protein kinase activity & $1.37 \mathrm{E}-03$ \\
\hline GO:0009742 & brassinosteroid mediated signaling & $7.15 \mathrm{E}-04$ \\
\hline GO:0009736 & cytokinin mediated signaling & $3.71 \mathrm{E}-07$ \\
\hline
\end{tabular}

MAPK/ERK Kinase may directly or indirectly act through another signaling cascade to activate a transcription factor. The transcription factor will then bind a particular region of DNA, resulting in the recruitment of RNA polymerase to transcribe a gene that will ultimately contribute to altering the function of the cell and cause pathogenesis[35]. These evidences in literature support our predicted results.

\section{Subcellular localization of Arabidopsis proteins targeted by the predicted Pseudomonas proteins}

Pathogens suppress host immunity by directing a range of secreted proteins or effectors, to the cytoplasm of host cells. Once these effector proteins traversed the host plasma-membrane, are transported to many subcellular locations where they subvert the host immune system to enable pathogen growth and reproduction. The knowledge of cellular compartments of the Arabidopsis proteins targeted by the predicted Pseudomonas will be helpful in deciphering the mechanism of host-pathogen interactions. If the targeted Arabidopsis proteins are located in cellular compartments that are very relevant to the pathogen's infection or very likely to be involved in interactions with the pathogen, then the prediction result supports the hostpathogen predictions.

To have a clear understanding the location of the interactions in host, we extracted the subcellular localization of the predicted Arabidopsis proteins from both the domain based and interolog methods using the AtSubP [36] available in TAIR database. To date, AtSubP is the only tool for subcellular location prediction of Arabidopsis proteins on a genome-scale with high accuracy for seven locations. The subcellular locations of all predicted Arabidopsis proteins are listed in Table 4 . We found that $29 \%$ host proteins are localized in nucleus, $9 \%$ in extracellular, $10 \%$ in chloroplast, $16 \%$

Table 3 Enriched GO biological process terms involved in predicted Pseudomonas syringae proteins

\begin{tabular}{lll}
\hline GO id & GO term & $p$-value \\
\hline GO:0006468 & protein amino acid phosphorylation & $1.70 \mathrm{E}-19$ \\
\hline GO:0018202 & peptidyl-histidine modification & $4.51-17$ \\
\hline GO:0006796 & phosphate metabolic process & $2.80 \mathrm{E}-15$ \\
\hline GO:0000160 & two-component signal transduction system & $3.62 \mathrm{E}-16$ \\
\hline GO:0006355 & regulation of transcription, DNA-dependent & $2.58 \mathrm{E}-04$ \\
\hline GO:0051252 & regulation of RNA metabolic process & $2.66 \mathrm{E}-04$ \\
\hline GO:0006935 & chemotaxis & $1.25 \mathrm{E}-03$ \\
\hline GO:0007626 & locomotory behavior & $1.25 \mathrm{E}-03$ \\
\hline GO:0018202 & peptidyl-histidine modification & $2.07 \mathrm{E}-03$ \\
GO:0009405 & pathogenesis & $7.11 \mathrm{E}-04$ \\
\hline GO:0006026 & aminoglycan catabolic process & $1.54 \mathrm{E}-03$ \\
\hline GO:0016998 & cell wall macromolecule catabolic process & $2.67 \mathrm{E}-03$ \\
\hline GO:0052034 & negative regulation by symbiont of pathogen-associated molecular pattern-induced host innate immunity & $5.03 \mathrm{E}-04$ \\
\hline GO:0000272 & polysaccharide catabolic process & $9.92 \mathrm{E}-03$ \\
\hline GO:0006022 & aminoglycan metabolic process & $4.37 \mathrm{E}-02$ \\
\hline GO:0040029 & regulation of gene expression, epigenetic & $1.65 \mathrm{E}-03$ \\
\hline GO:0016458 & gene silencing & $1.32 \mathrm{E}-02$ \\
\hline GO:0075343 & negative regulation by symbiont of defense-related host callose deposition \\
\hline GO:0006342 & chromatin silencing & $1.65 \mathrm{E}-03$ \\
\hline
\end{tabular}


Table 4 Distribution of subcellular localization for predicted interacting proteins in Arabidopsis thaliana from both the domain and interolog-based approaches

\begin{tabular}{ccc}
\hline Arabidopsis protein location & \# of proteins & Percentage (\%) \\
\hline Nucleus & 4101 & 29 \\
\hline Chloroplast & 1356 & 10 \\
\hline Cytoplasm & 2317 & 16 \\
\hline Golgi apparatus & 192 & 1 \\
\hline Mitochondrion & 770 & 5 \\
\hline Extracellular & 1199 & 9 \\
\hline Cell membrane & 1321 & 10 \\
\hline Unknown & 2787 & 20 \\
\hline
\end{tabular}

* Predictions were generated using Arabidopsis-specific subcellular localization predictor, AtSubP (Plant Physiology 2010, 154: 36-54).

in cytoplasm, $10 \%$ in cell membrane, $1 \%$ in Golgi, $5 \%$ in mitochondrion and $20 \%$ as unknown. It reveals that major of the interactions occur in nucleus, cytoplasm, chloroplast and plasma membrane region. In a recent review by Block and James [33] shows that the effectors of Pseudomonas syringae target the plant proteins mostly in plasma membrane, chloroplast and mitochondrion. Citovsky et al. [37] showed that when Agrobacterium tumefaciens interact with $A$. thaliana, it hijacks VIP1 protein and use it to shuttle transfer-DNA (T-DNA) into the nucleus for its reproduction. Tao et al. investigated that TIP, an Arabidopsis protein, interacts with the coat protein (CP) of Turnip crinkle virus (TCV) in yeast cells in nuclei [38]. Thus, the predicted locations of the interacting Arabidopsis proteins by our approach are in close agreement with the earlier findings. Also the localizations for a large number of proteins are still unknown which need a special attention for experimental characterization.

\section{Conclusion}

In this study, we have demonstrated that the sequence and domain similarity to known interactions are valuable information in predicting the host-pathogen interactions. We identified 11000 PPIs between Arabidopsis thaliana and Pseudomonas syringae pv. tomato DC3000 based on the domain-based and interolog approaches. The functional annotations of both Arabidopsis and Pseudomonas proteins involved in the predicted PPI are analyzed and it shows the relevance of the proteins for host defense and pathogen infections. The present work may provide some useful information and resource to the plant community to understand the molecular mechanism of the plant immunity system against pathogen virulence. The quality of the predicted interactome could further be improved by combining these methods with other computational approaches and biological data sources. The reliability of the predicted interactions can be further assessed through experimental validations.

\section{Additional material}

Additional file 1: Predicted protein-protein interaction pairs for Arabidopsis-Pseudomonas syringae based on the domain model.

Additional file 2: Predicted protein-protein interaction pairs for Arabidopsis-Pseudomonas syringae based on the interolog model.

Additional file 3: Consensus protein-protein interaction pairs predicted based on both the domain and interolog models in ArabidopsisPseudomonas syringae.

\section{List of abbreviations used}

Pst DC3000, Pseudomonas syringae pathovar tomato DC3000; DIP, Database of Interacting Proteins; HPIDB, Host Pathogen Interaction Database; PPI, protein-protein interaction; GO, Gene Ontology; TTSS, type III protein secretion system; 3DID, three-dimensional interacting domains; iPfam, Protein family interactions; DAVID, Database for Annotation, Visualization and Integrated Discovery.

\section{Competing interests}

The authors declare that they have no competing financial interests.

\section{Authors' contributions}

SSS collected the host and pathogen proteome datasets, developed algorithms and models, performed the calculations, figures and tables, and wrote the draft manuscript. TW helped in data analysis and setting up the pipelines on High-Performance Computing Center. RK conceived the study, participated in its design and coordination, and edited the final manuscript. All authors read and approved the final manuscript.

\section{Acknowledgements}

The authors duly acknowledge the funding support to this study from Oklahoma Center for Advancement of Science and Technology (OCAST), grant number PS12-062. We also thank the anonymous referees for critical review of the manuscript that helped improving the research article.

\section{Declaration}

Funding for the publication of this article has come from OCAST funds account AB-5-42820, OSU.

This article has been published as part of BMC Bioinformatics Volume 15 Supplement 11, 2014: Proceedings of the 11th Annual MCBIOS Conference. The full contents of the supplement are available online at http://www. biomedcentral.com/bmcbioinformatics/supplements/15/S11.

\section{Authors' details}

${ }^{1}$ National Institute for Microbial Forensics \& Food and Agricultural Biosecurity (NIMFFAB), Oklahoma State University, Stillwater, 74078, USA. ${ }^{2}$ Department of Biochemistry \& Molecular Biology, Oklahoma State University, Stillwater, 74078, USA. ${ }^{3}$ Bioinformatics Facility, Department of Botany \& Plant Sciences, Institute for Integrative Genome Biology (IIGB), University of California, Riverside, California, 92521, USA.

Published: 21 October 2014

\section{References}

1. Katagiri F, Thilmony R, SY H: The Arabidopsis thaliana-Pseudomonas syringae Interaction. The Arabidopsis Book, Rockville, MD, USA: American Society of Plant Biologists 2002, 11-35.

2. Barah $P$, Winge $P$, Kusnierczyk A, Tran DH, Am B: Molecular signature of Arabidopsis thaliana in response to Insect attack and bacterial attack. PLOS One 2013, 8(3):e58987.

3. Abramovitch RB, Gb M: Strategies used by bacterial pathogens to suppress plant defenses. Curr Opin Plant Biol 2004, 7(4):356-364.

4. Quirino BF, Af B: Deciphering host resistance and pathogen virulence: the Arabidopsis/Pseudomonas interaction as a model. Molecular Plant Pathology 2003, 4(6):517-530.

5. Buell CR, Joardar V, Lindeberg M, Selengut J, Paulsen IT, Gwinn ML, Dodson RJ, Deboy RT, Durkin AS, Jf K, et al: The complete genome 
sequence of the Arabidopsis and tomato pathogen Pseudomonas syringae pv. tomato DC3000. Proc Natl Acad Sci 2003, 100(18):10181-10186

6. Nomura K, Melotto M, Sy H: Suppression of host defense in compatible plant-Pseudomonas syringae interactions. Curr Opin Plant Biol 2005, 8(4):361-368

7. Bishop JG, Dean AM, T M-O: Rapid evolution in plant chitinases: molecular targets of selection in plant-pathogen coevolution. Proc Natl Acad Sci USA 2000, 97(10):5322-5327.

8. Mukhtar MS, Carvunis AR, Dreze M, Epple P, Steinbrenner J, Moore J, Tasan M, Galli M, Hao T, Nishimura MT, et al: Independently evolved virulence effectors converge onto hubs in a plant immune system network. Science 2011, 333(6042):596-601.

9. Goritschnig S, Krasileva KV, Dahlbeck D, Bj S: Computational Prediction and Molecular Characterization of an Oomycete Effector and the Cognate Arabidopsis Resistance Gene. PLOS genetics 2012, 8(2):e1002502.

10. Pinzon A, Rodriguez RL, Gonzalez A, Bernal A, S R: Targeted metabolic reconstruction: a novel approach for the characterization of plantpathogen interactions. Brief Bioinform 2011, 12(2):151-162.

11. Kim JG, Park D, Kim BC, Cho SW, Kim YT, Park YJ, Cho HJ, Park H, Kim KB, Yoon KO, et al: Predicting the interactome of Xanthomonas oryzae pathovar oryzae for target selection and db service. BMC Bioinformatics 2008, 9:41

12. Wu X, Zhu L, Guo J, Zhang DY, K L: Prediction of yeast protein-protein interaction network: insights from the gene ontology and annotations. Nucleic Acids Research 2006, 34(7):2137-2150.

13. Matthews LR, Vaglio P, Reboul J, Ge H, Davis BP, Garrels J, Vincent S, M V: Identification of potential interaction networks using sequence-based searches for conserved protein-protein interactions or "interologs". Genome Research 2001, 11(12):2120-2126.

14. Ng SK, Zhang Z, SH T: Integrative approach for computationally inferring protein domain interactions. Bioinformatics 2003, 19(8):923-929.

15. Ogmen U, Keskin O, Aytuna AS, Nussinov R, A G: Prism: protein interactions by structural matching. Nucleic Acids Research 2005, 33: W331-W336.

16. Davis FP, Barkan DT, Eswar N, Mckerrow JH, A S: Host-Pathogen protein interactions predicted by comparative modeling, Protein Science. Protein Science 2007, 16(12):2585-2596.

17. Shoemaker BA, Ar P: Deciphering protein-protein interactions. Part ii. Computational methods to predict protein and domain interaction partners. PLoS Comput Bio 2007, 3(4):e43.

18. Li ZG, He F, Zhang Z, YL P: Prediction of protein-protein interactions between Ralstonia solanacearum and Arabidopsis thaliana. Amino Acids 2012, 42:2363-2371.

19. Kurubanjerdjit N, Tsai JJP, Sheu CY, KI N: The prediction of protein-protein interaction of A. thaliana and X. campestris pv. campestris based on protein domain and interolog approaches. Plant Omics Journal 2013, 6(6):388-398.

20. Schlekera S, Garcia-Garciab J, Seetharamana JK, B O: Prediction and comparison of Salmonella-human and Salmonella-Arabidopsis interactomes. Chem Biodivers 2012, 9(5):991-1018.

21. Zhou H, Rezaei J, Hugo W, Gao S, Jin J, Fan M, Yong CH, Wozniak M, L W: Stringent DDI-based Prediction of H. sapiens-M. tuberculosis H37Rv Protein-Protein Interactions. BMC Systems Biology 2013, 7(6):S6.

22. S W: Computational Prediction of Host-Parasite Protein Interactions between P. falciparum and H. sapiens. PLOS ONE 2011, 6(11):e26960

23. Dyer $M$, et al: Computational prediction of host-pathogen protein-protein interactions. Bioinformatics 2007, 23:1159-i166.

24. Xenarios I, Salwinski L, Duan XJ, Higney P, Kim S, D E: DIP: The Database of Interacting Proteins. A research tool for studying cellular networks of protein interactions. Nucleic acids research 2002, 30:303-305.

25. Kumar R, B N: HPIDB-a unified resource for host-pathogen interactions. BMC Bioinformatics 2010, 11:S16.

26. Yu NY, Wagner JR, Laird MR, Melli G, Rey S, Lo R, Dao P, Sahinalp SC, Ester M, Foster $L$, et al: PSORTb 3.0: Improved protein subcellular localization prediction with refined localization subcategories and predictive capabilities for all prokaryotes. Bioinformatics 2010, 26(13):1608-1615.

27. Jehl MA, Arnold R, T R: Effective - a database of predicted secreted bacterial proteins. Nucleic Acids Research 2010, 1-5.
28. Quevillon E, et al: InterProScan: protein domains identifier. Nucleic Acids Research 2005, 33:W116-W120.

29. Yu H, Luscombe NM, L H, et al: Annotation Transfer Between Genomes: Protein-Protein Interologs and Protein-DNA Regulogs. Genome Research 2004, 14:1107-1118.

30. Huang DW, Sherman BT, RA L: Systematic and integrative analysis of large gene lists using DAVID Bioinformatics Resources. Nature Protocol 2009, 4(1):44-57.

31. Fones $\mathrm{H}$, et al: Metal hyperaccumulation armors plants against disease. PLoS Pathogen 2010, 6(9):p1.

32. Franza $T$, Mahe $B, D$ E: Erwinia chrysanthemi requires a second iron transport route dependent of the siderophore achrophore achromobactin for extracellular growth and plant infection. Mol Microbiol 2005, 55:261-275.

33. Block A, Jr A: Plant targets for Pseudomonas syringae type III effectors: virulence targets or guarded decoys? Current Opinion in Microbiology. Current Opinion in Microbiology 2011, 14:39-46.

34. Qiu JL, Fiil BK, Petersen K, Nielsen HB, Botanga CJ, Thorgrimsen S, Palma K Suarez Rodriguez MC, Sandbech Clausen S, Lichota J, et al: Arabidopsis MAP kinase 4 regulates gene expression through transcription factor release in the nucleus. European Molecular Biology Organization Journal 2008, 27:2214-2221.

35. Ligterink W, Kroj T, zurNieden U, Hirt H, D S: Receptor-mediated activation of a MAP kinase in pathogen defense of plants. Science 1997, 276(5321):2054-2057.

36. Kaundal R, Saini R, PX Z: Combining Machine Learning and HomologyBased Approaches to Accurately Predict Subcellular Localization in Arabidopsis. Plant Physiology 2010, 154:36-54.

37. Citovsky V, Kapelnikov A, Oliel S, Zakai N, Rojas MR, Gilbertson RL, Tzfira T, A L: Protein interactions involved in nuclear import of the Agrobacterium VirE2 Protein in vivo and in vitro. Journal of Biological Chemistry 2004, 279:29528-29533.

38. Tao R, Qu F, TJ M: The Nuclear Localization of the Arabidopsis Transcription Factor TIP Is Blocked by Its Interaction with the Coat Protein of Turnip Crinkle Virus. Virology 2005, 331(2):316-324.

doi:10.1186/1471-2105-15-S11-S13

Cite this article as: Sahu et al:: Predicting genome-scale Arabidopsis-

Pseudomonas syringae interactome using domain and interolog-based approaches. BMC Bioinformatics 2014 15(Suppl 11):S13.

\section{Submit your next manuscript to BioMed Central and take full advantage of:}

- Convenient online submission

- Thorough peer review

- No space constraints or color figure charges

- Immediate publication on acceptance

- Inclusion in PubMed, CAS, Scopus and Google Scholar

- Research which is freely available for redistribution

Submit your manuscript at www.biomedcentral.com/submit
Biomed Central 